\title{
Curved reformat of the paediatric brain into a 'Mercator/flat-earth map' - A standardised method for demonstrating cortical surface atrophy resulting from hypoxic ischaemic injury
}

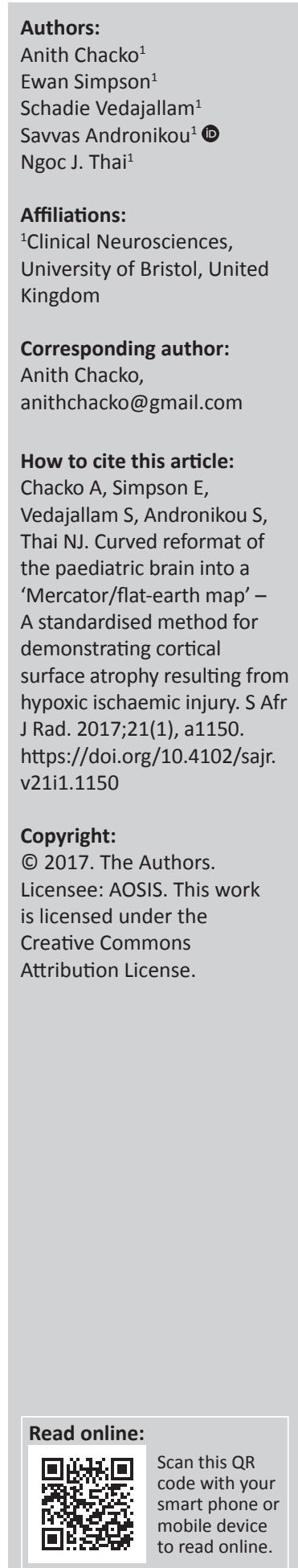

Magnetic resonance imaging (MRI) in term hypoxic ischaemic injury (HII) demonstrates cortical atrophy in characteristic locations. Communicating bilateral zonal injury to parents and in courtrooms using reports and cross-sectional imaging is challenging. An overview map of the brain surface generated from a curved reconstruction of the MRI is ideal for such scenarios. Freeware was used to derive standardised methods of curved reconstructions of the paediatric brain from three-dimensional MRI in 10 children with cortical atrophy from term HII and 10 agematched 'controls'. Multiple techniques were tested from various planes, different landmark slices and angles of reconstruction at various depths to the surface. Two images (derived from the coronal and sagittal planes) were identified as giving the best overview of the watershed, perisylvian and peri-rolandic zones. Peri-sylvian, peri-rolandic zones and frontal lobes were better demonstrated on Mercator maps. Scroll maps demonstrated lateral structures poorly but demonstrated central zones, posterior parietal lobes and occipital lobes better. Watershed zones were well demonstrated on both maps. Localised 'bi-convex' interhemispheric fissure widening was present in all HII atrophy patients on both maps, that is, para-sagittal and/or para-falcine watershed atrophy. An intervascular watershed band of atrophy was seen on both maps. Perirolandic and peri-sylvian atrophy was better demonstrated on Mercator maps. Ulegyria was identified in $90 \%$. Standardised curved reconstruction of the brain surface from MRI allows visualisation of key cortical features of term HII on the two derived images which can replace multi-slice MRI for communicating cortical findings to legal professionals and parents, and can be embedded within multimedia reports.
Note: A selection of conference abstracts: RSSA/SASPI Paediatric Imaging Congress, 03-06 November 2016, Spier Estate, Stellenbosch, South Africa. Faculty collaborators: Professor Kassa Darge (Body Imaging, University of Pennsylvania, Philadelphia, USA), Professor Edward Lee (Thoracic Imaging, Harvard University, USA), Professor Beverley Newman (Cardiac Imaging, Stanford University, California, USA), Professor Kimberly Applegate (Image Gently and Body Imaging, Emory University, Atlanta, USA) and Professor Savvas Andronikou (Thoracic Imaging, University of Bristol, UK) supported by South African Paediatric Radiologists, co-ordinated by Dr. Jaishree Naidoo, President of the African Society of Paediatric Imaging and Head of Division of Paediatric Radiology, Charlotte Maxeke Johannesburg Academic Hospital.

This conference abstract is partially based on the following publication: Society of Pediatric Radiology, Reston, VA (United States) (2016). IPR 2016 Abstracts. Pediatric Radiology, 46(Suppl1), 1-372. https://doi.org/10.1007/s00247-016-3579-x 